\title{
Modeling of Thermodiffusion Inertia in Metal Films Heated with Ultrashort Laser Pulses
}

\author{
A.J. JANAVIČIUS ${ }^{a}$ AND S. TuRskien: $\dot{\mathrm{E}}^{b}$ \\ ${ }^{a}$ Faculty of Natural Sciences, Šiauliai University \\ Višinskio 19, Šiauliai, 76351, Lithuania \\ ${ }^{b}$ Faculty of Mathematics and Informatics, Šiauliai University \\ Višinskio 19, Šiauliai, 76351, Lithuania
}

(Received June 26, 2006)

\begin{abstract}
Previously, we considered the analytical solution of nonlinear diffusion equation in two-dimensional surface. This allows us to consider the same nonlinear heat conduction equation for metals heated by a picosecond laser. After fast thermalization (within a few femtoseconds) of the laser energy and electron excitation in the conductivity band, electrons ballistically diffuse and transfer their energies to the target atoms and equilibrium electrons from the conductivity band. In this case, we have thermal diffusion of nonequilibrium excited electrons and thermal inertia for thermodiffusion. The relaxation time of excited conductivity electrons is directly connected with thermal inertia and depends on electron-electron collision frequencies. The theoretical temperature surface plots for the thermodiffusion of excited electrons were obtained from analytical solutions of nonlinear thermodiffusion equations.
\end{abstract}

PACS numbers: 66.30.-h, 79.20.Ds

\section{Introduction}

The conventional linear diffusion and heat transport equations have the same form and we can suppose that for nonlinear diffusion equations [1, 2] situation is the same. Taking into account that the diffusion velocity and the maximum penetration depth of impurity atoms must be finite, the following nonlinear mass flux and diffusion equations was proposed in an ancient paper [3]:

$$
j(x, t)=-D_{n} n(x, t) \frac{\mathrm{d}}{\mathrm{d} x} n(x, t),
$$




$$
\begin{aligned}
& \frac{\mathrm{d}}{\mathrm{d} t} n(x, t)=D_{n} \frac{\mathrm{d}}{\mathrm{d} x}\left[n(x, t) \frac{\mathrm{d}}{\mathrm{d} x} n(x, t)\right], \\
& D(x, t)=D_{n} n(x, t) .
\end{aligned}
$$

Here, the diffusion coefficient $D$ is directly proportional to concentration of impurities $n(x, t)$. The proposed model includes the realistic assumption according to which the mass flux (1) $j$ at the point $x+\Delta x$ differs from zero only when the impurity gradient and concentration are different from zero at the point $x$. We can rewrite the expression for the mass flux (1) $j$ using finite difference scheme

$$
j(x+\Delta x, t) \approx-D_{n} n(x, t) \frac{n(x+\Delta x, t)-n(x, t)}{\Delta x} .
$$

The presented equation means that the jump of diffusing particles from point $x$ to $x+\Delta x$ is possible only when the diffusing particle exists in the point $x$. We assume that the jump length of diffusing particles in solids, fluids or the mean free path in a gas have finite value. In the case of heat transfer by electrons, the situation is similar. The maximum penetration depth of diffusing impurity atoms or heat transferring electrons must consist of finite frequency of atoms jumps or scattering frequencies of electrons and depends on diffusion or thermodiffusion coefficients. Any jump of a diffusing atom or electron-electron and electron-phonon scattering occurs during a definite time interval. The velocity of the nonlinear diffusion and thermodiffusion must be finite. The very fast diffusion or superdiffusion of indium atoms in $\mathrm{HgCdTe}$ crystal [4] activated by tungsten-halogen radiation and very fast diffusion of vacancies generated by soft X-rays in Si crystals [5] were realized experimentally and successfully explained theoretically $[6,7]$ using nonlinear diffusion Eq. (1), for two-level excited systems when the Boltzmann distribution is valid. The diffusion coefficient (3) depends on relaxation time of population [5] $P(t)$ of the excited state by fast diffusing particles

$$
D(x, t)=D_{n} n(x, t), \quad D_{n}=\frac{D_{0}}{n(0, t) \alpha} P(t), \quad P(t)=\left(\frac{t}{t_{0}}\right)^{\alpha-1} .
$$

Here $D_{0}$ is pre-exponential factor of diffusion coefficient for non-excited system, $\alpha$ - excitation parameter and $t_{0}$ - relaxation time of excited system. When excitation parameter $\alpha$ tends to zero, we have superdiffusion [6]. We used in this work the obtained results for fast thermodiffusion of electrons in metals heated by picosecond laser pulses.

We will use the two temperature model [8] based on coupled temperature evolution of electronic and atomic subsystems and proposition that conductivity and thermodiffusion is coursed only by excited nonequilibrium electrons [9].

\section{Nonlinear heat conduction equation for excited systems}

Heat conduction process can be viewed as diffusion of more energetic particles. We can express the heat amount transferred through surface $\Delta S$ during time $\mathrm{d} t$ according to (1) in the second way 


$$
\mathrm{d} Q=\Lambda_{\mathrm{e}}\left[T_{\mathrm{d}} \frac{\mathrm{d} T_{\mathrm{d}}}{\mathrm{d} x}+\mathrm{d}\left(T_{\mathrm{d}} \frac{\mathrm{d} T_{\mathrm{d}}}{\mathrm{d} x}\right)\right] \Delta S \mathrm{~d} t-\Lambda_{\mathrm{e}} T_{\mathrm{d}} \frac{\mathrm{d} T_{\mathrm{d}}}{\mathrm{d} x} \Delta S \mathrm{~d} t, \quad T_{\mathrm{d}}=T_{\mathrm{e}}-T_{\mathrm{a}},(6)
$$

where $\Lambda_{\mathrm{ne}}=\Lambda_{\mathrm{e}} / T_{\mathrm{e}}(0, t)$ is nonlinear heat conductivity coefficient, $T_{\mathrm{e}}$ and $T_{\mathrm{a}}$ are temperatures of more energetic particles (gas of conductivity electrons in metals excited by laser irradiation) and the less energetic particles (non-excited electrons and atoms of the lattice) consequently. During a collision process, the less energetic particles gain the energy

$$
\mathrm{d} Q=\left(C_{\mathrm{e}} \mathrm{d} T_{\mathrm{e}}+C_{\mathrm{a}} \mathrm{d} T_{\mathrm{a}}\right) \Delta A \mathrm{~d} x,
$$

where for copper [10] $C_{\mathrm{e}}=\gamma T_{\mathrm{e}}, \gamma=96.6 \mathrm{~J} /\left(\mathrm{m}^{3} \mathrm{~K}^{2}\right)$ and $C_{\mathrm{a}}=3.5 \times 10^{6} \mathrm{~J} /\left(\mathrm{m}^{3} \mathrm{~K}\right)$ denotes the specific heats of electrons and atoms systems. Usually, the heat transfer from excited electrons to the atomic system can be described by the heat conduction equation [10]:

$$
C_{\mathrm{a}} \frac{\mathrm{d} T_{\mathrm{a}}}{\mathrm{d} t}=g\left(T_{\mathrm{e}}-T_{\mathrm{a}}\right)
$$

where electron-phonon coupling constant $g=10^{17} \mathrm{~W} /\left(\mathrm{K} \mathrm{m}^{3}\right)$. We will represent the source term like in [10]:

$$
\begin{aligned}
& Q(x, t)=\frac{\Phi_{\mathrm{a}}}{\tau \lambda} \mathrm{e}^{-x / \lambda}, \quad \Phi_{\mathrm{a}}=(1-R) \Phi, \quad 0<t<\tau, \\
& E=\Phi_{\mathrm{a}} \frac{\Delta t}{\tau} \Delta S, \quad \Delta S=2.169 \times 2.169 \mathrm{~nm}^{2} .
\end{aligned}
$$

Here $\lambda=14 \mathrm{~nm}$ denotes the laser penetration depth, short laser pulse length $\tau=0.5 \mathrm{ps}, \Delta t$ - laser pulse duration, $\Delta S$ - irradiated square area, $\Phi$ is laser heating pulse fluence with the energy $E=3.2 \times 10^{-15} \mathrm{~J} / \mathrm{nm}^{2}$. Here we choose $\Phi=170 \mathrm{~mJ} / \mathrm{cm}^{2}, R=0.6$ is the target reflection coefficient. By including (9) into (6) and comparing (6) with (7) we obtain nonlinear heat conduction equation

$$
C_{\mathrm{e}} \frac{\mathrm{d} T_{\mathrm{d}}}{\mathrm{d} t}=\Lambda_{\mathrm{ne}} \frac{\mathrm{d}}{\mathrm{d} x}\left(T_{\mathrm{d}} \frac{\mathrm{d} T_{\mathrm{d}}}{\mathrm{d} x}\right)-\left(\frac{C_{\mathrm{e}}}{C_{\mathrm{a}}}+1\right) g T_{\mathrm{d}}+Q(x, t)
$$

for the metals heated by laser. We will use a similar equation for the two-dimensional case

$$
\begin{aligned}
\frac{\mathrm{d} T_{\mathrm{d}}}{\mathrm{d} t} & =\frac{\Lambda_{\mathrm{ne}}}{C_{\mathrm{e}}}\left[\frac{\mathrm{d}}{\mathrm{d} x}\left(T_{\mathrm{d}} \frac{\mathrm{d} T_{\mathrm{d}}}{\mathrm{d} x}\right)+\frac{\mathrm{d}}{\mathrm{d} y}\left(T_{\mathrm{d}} \frac{\mathrm{d} T_{\mathrm{d}}}{\mathrm{d} y}\right)\right] \\
& -\left(\frac{1}{C_{\mathrm{a}}}+\frac{1}{C_{\mathrm{e}}}\right) g T_{\mathrm{d}}+\frac{1}{C_{\mathrm{e}}} Q(x, y, t) .
\end{aligned}
$$

The heat source term $Q(x, y, t)$ can be included as the first stage [11] of thermal diffusion. We use the approximation [12] where electron-phonon coupling constant $g$ for subpicosecond scale of time can be neglected. Thermalization of the laser energy in the conduction band continues for a few femtoseconds [10] and heat transporting excited electrons moves with velocity close to the Fermi velocity, which in the case of copper is $v_{\mathrm{F}}=1.57 \times 10^{3} \mathrm{~nm} / \mathrm{ps}$. In the first stage of heat transport by excited hot electrons, which are out equilibrium with the lattice, it must be taken into account that the delay in heat transport by electrons increases 
linearly with the sample thickness [12]. In this case (5) the excitation parameter [11] for thermodiffusion coefficient

$$
D_{\mathrm{t}}=\frac{D_{0 \mathrm{t}}}{\alpha} P(t), \quad D_{0 \mathrm{t}}=\frac{\Lambda_{\mathrm{e} 0}}{C_{\mathrm{e} 0}}, \quad P(t)=\left(\frac{t}{t_{0}}\right)^{\alpha-1}, \quad \Lambda_{0}=3.85 \times 10^{-19}
$$

must be $\alpha=2$. Here [10] $\Lambda_{\mathrm{e} 0}=4 \times 10^{-19} \mathrm{~J} /(\mathrm{ps} \mathrm{nm} \mathrm{K})$ and $C_{\mathrm{e} 0}=\gamma T_{\mathrm{e} 0}$ $\left(\gamma=96.6 \times 10^{-27} \mathrm{~J} /\left(\mathrm{nm}^{3} \mathrm{~K}\right)\right)$ are the lattice heat conductivity and electronic specific heat at room temperature $T_{\mathrm{e} 0}=300 \mathrm{~K}$ for copper. We can evaluate this parameter using maximum distance $x_{0}$ traveled by hot electrons from the heat source [13], propagating by random walk or diffusion way in a two-dimensional surface

$$
x_{0}=0.632 \sqrt{D_{\mathrm{t}} t} .
$$

Comparing it with (12) we obtain

$$
x_{0}=0.632 \sqrt{\frac{D_{0 \mathrm{t}}}{\alpha} t_{0}^{1-\alpha}} t^{\frac{\alpha}{2}} .
$$

Requiring that the distance traveled by the excited electrons is proportional to time, we suppose that thermodiffusion wave in copper propagates with the constant speed of the same magnitude like the Fermi velocity $v_{\mathrm{F}}=1.57 \times 10^{6} \mathrm{~m} / \mathrm{s}$. Taking into account that in this case electrons move ballistically

$$
v_{\mathrm{F}}=0.632 \sqrt{\frac{D_{0 \mathrm{t}}}{\alpha} t_{0}^{1-\alpha}}, \quad \alpha=2
$$

and using (12) we can obtain thermalization time $t_{0}=\tau_{1}$ for electrons

$$
\tau_{1}=1.118 \times 10^{-15} \mathrm{~s} .
$$

This result is in accordance with the statement in paper [10] that fast thermalization of the laser energy in the conduction band electrons occurs in a few femtoseconds. During this very short thermalization time $t_{0}$, excited electrons move ballistically achieving distance $x_{0}=3.512 \mathrm{~nm}$ practically without any transfer of their energy to the target atoms [12]. In this first stage of heat transfer, when $\alpha=2$, we have ballistic thermodiffusion. After a few collisions with electrons of conductivity band and Coulomb's interaction with ions of lattice, ballistic electrons lose some amount of their energy. Further on, they participate in the second stage of thermodiffusion. For this second stage of fast thermodiffusion of hot electrons we have [11] $\alpha<1$ with thermodiffusion coefficient decreasing on time

$$
D_{\mathrm{t}}=\frac{D_{0 \mathrm{t}}}{\alpha}\left(\frac{t}{t_{0}}\right)^{\alpha-1}
$$

When $t=t_{0}$, we can find the excitation parameter $\alpha[6]$ for thermodiffusion process

$$
\alpha=\frac{D_{0 \mathrm{t}}}{D_{\mathrm{t}}}
$$

using the diffusion coefficients for excited $D_{\mathrm{t}}$ and for non-excited $D_{0 \mathrm{t}}$ or weakly 
excited system. Thus, after a short time of ballistic thermodiffusion (first stage of thermodiffusion) electrons heated by laser waste their energy in the second stage of thermodiffusion. In this second stage electrons diffuse very quickly transporting their energy to the conductivity band electrons [12]. This second stage of thermodiffusion can be characterized by excitation parameter $\alpha<1$ and thermodiffusion coefficient (17), which decreases with time. Both these kinds of diffusion were considered in the paper for explanation superdifusion of atoms $[11,14]$ and vacancies $[14,5]$ in excited crystals. Also in the paper [11] it was shown that for excitation parameter $\alpha<1$ the diffusion profile is only weakly time-dependent and we have some inertia and impurity localization in diffusion phenomenon. The similar thermal inertia were obtained and considered by solving hyperbolic heat conduction equation [12] for thermodiffusion of electrons and thermal energy localization in Au film heated by the ultrashort laser pulse. The diffusion (5) or thermodiffusion (11) coefficients are defined by time dependence of ratio of two energy levels $P(t)$ [11]. The very fast thermodiffusion in the second stage is related to decreasing $P(t)$ or number of ballistically moving electrons due to collisions with conductivity band electrons. This process can be characterized by some relaxation time $t_{0}=\tau_{2}$. This relaxation time depends on metal and also on electron-electron collisions frequency and energy of ballistic electrons. Using the thermodiffusion coefficient (12), which depends on the thermalization time and population of excited states, we can say that presented nonlinear Eq. (10) describes the heat transfer by ballistic electrons and the thermodiffusion of the excited electrons. In this case or first stage of thermodiffusion we have nearly ballistic heat transport for this very short time. For the first and second stages of thermodiffusion we can use approach $g=0$ [12]. The second stage of fast thermodiffusion is characterized by decreasing population of electrons in excited states due to the heat transporting process. The population decrease is defined by electron's excitation parameter $\alpha$ and relaxation time $t_{0}=\tau_{2}$. Instead of electron-electron interaction parameters we can use, as in [12], the relaxation time $t_{0}=\tau_{2}$ and additionally the excitation parameter $\alpha$.

\section{Calculation of thermodiffusion in thin metal's films}

Laser light is absorbed in metals by the conduction-band electrons within a few femtoseconds [10]. In this first stage electrons move ballistically without electron-electron and without electrons collisions. Usually the temporal shape of laser pulse is taken as Gaussian. We can approximate the shape of energy deposition transmitted to the electrons by this pulse in the plane with modified $Q(x, y, t)$ for two dimensions [10]. We can describe this first stage of thermodiffusion taking $g=0$ in Eq. (11). Using the solution of the similar nonlinear equation for diffusion in the plane [13], we can present the approximate analytical solution of nonlinear thermodiffusion Eq. (11) in the following way:

$$
T_{\mathrm{d} 1}(x, y, t)=T_{\mathrm{s} 1} f\left(x_{1}, y_{1}\right), \quad T_{\mathrm{s} 1}=T_{\mathrm{s} 1}(0,0, t)-T_{\mathrm{a}}(0,0, t),
$$




$$
\begin{aligned}
& x_{1}=-0.632+\frac{|x|}{\sqrt{D t}}, \quad y_{1}=-0.632+\frac{|y|}{\sqrt{D t}}, \\
& f\left(x_{1}, y_{1}\right)=-0.316 x_{1}-0.316 y_{1}-0.5 x_{1}^{2}-0.5 y_{1}^{2}+2.5 x_{1} y_{1}, \\
& -R_{0} \leq x \leq R_{0}, \quad-R_{0} \leq y \leq R_{0}, \quad R_{0}=0.632 \sqrt{D t} .
\end{aligned}
$$

Here $T_{\mathrm{s} 1}(0,0, t)$ and $T_{\mathrm{a}}(0,0, t)$ are electrons and lattice temperatures in the center of irradiated plane, $D$ - thermodiffusion coefficient.

We can compare the irradiated energy $E$ by laser [10] and energy absorbed by conductivity electrons in the area $\Delta S=2.169 \times 2.169 \mathrm{~nm}^{2}$, using the known laser penetration depth $\lambda=14 \mathrm{~nm}$. For the laser with intensity $\Phi_{\mathrm{a}}=\Phi(1-R)$ and the source term

$$
Q(x, t)=\frac{\Phi_{\mathrm{a}}}{\tau \lambda} \exp (-x / \lambda)
$$

with parameters $\Phi=170 \mathrm{~mJ} / \mathrm{cm}^{2}, R=0.6, \tau=0.5 \mathrm{ps}$, we obtained the irradiated energy

$$
E=3.20 \times 10^{-15} \mathrm{~J}
$$

The average temperature of electrons in the irradiated volume $V=\Delta S \lambda$ of copper was obtained requiring that $E$ must be equal to the absorbed energy by conductivity electrons

$$
T_{\mathrm{s} 1}=\sqrt{\frac{2 E}{\gamma V}}, \quad T_{\mathrm{s} 1}=3.17 \times 10^{4} \mathrm{~K} .
$$

Taking into account that the temporal shape of laser pulse is Gaussian we can suppose that distribution of the excited electrons will be symmetric and even at the first moment of thermalization can be represented as the solution (19)

$$
T_{1}(x, y, t)=T_{\mathrm{s} 1} f\left(x_{1}, y_{1}\right), \quad T_{\mathrm{s} 1}=T_{\mathrm{e} 1}(0,0, t), \quad 0<t<\tau
$$

of thermodiffusion equation

$$
\frac{\mathrm{d} T_{\mathrm{d}}}{\mathrm{d} t}=\frac{\Lambda_{\mathrm{e}}}{C_{\mathrm{e}}}\left[\frac{\mathrm{d}}{\mathrm{d} x}\left(T_{\mathrm{d}} \frac{\mathrm{d} T_{\mathrm{d}}}{\mathrm{d} x}\right)+\frac{\mathrm{d}}{\mathrm{d} y}\left(T_{\mathrm{d}} \frac{\mathrm{d} T_{\mathrm{d}}}{\mathrm{d} y}\right)\right], \quad D=\frac{\Lambda_{\mathrm{e}}}{C_{\mathrm{e}}}
$$

for constant source (21), (22) for the irradiation time $t=\tau$. Using the presented solution (23) for the case $T_{\mathrm{s} 1}=$ const we can find the introduced thermal energy into conductivity electrons

$$
\begin{aligned}
\mathrm{d} Q_{\mathrm{e} 1} & =\gamma L \mathrm{~d} S T_{\mathrm{e}} \mathrm{d} T_{\mathrm{e}}, \\
Q_{\mathrm{el}}= & \gamma V T_{\mathrm{s} 1}^{2} 4 L \int_{-0.632}^{0} \mathrm{~d} x_{1} \int_{-0.632}^{0} \mathrm{~d} y_{1} f\left(x_{1}, y_{1}\right) \mathrm{d} f\left(x_{1}, y_{1}\right) \\
& =0.09135 D t_{1} L \gamma T_{\mathrm{s} 1}^{2} .
\end{aligned}
$$

By comparing the introduced thermal energy (25) $Q$ calculated from the thermodiffusion profile to the introduced energy (21) $E$ we can obtain ballistic thermodiffusion coefficient for process of excitation [6] of electrons in the conductivity band with excitation parameter (17) $\alpha=2$ : 


$$
D \tau_{1}=25.75 \mathrm{~nm}^{2}, \quad D_{1}=2.302 \times 10^{4} \frac{\mathrm{nm}^{2}}{\mathrm{ps}} .
$$

By replacing $D \tau_{1}$ with $D t$ in (19) we obtain the temperature profile (23), which is presented in Fig. 1. The lines of equal temperatures on the plane presented in Fig. 2 are shown for the case of irradiated conductivity electrons after the thermalization time $\tau_{1}$.

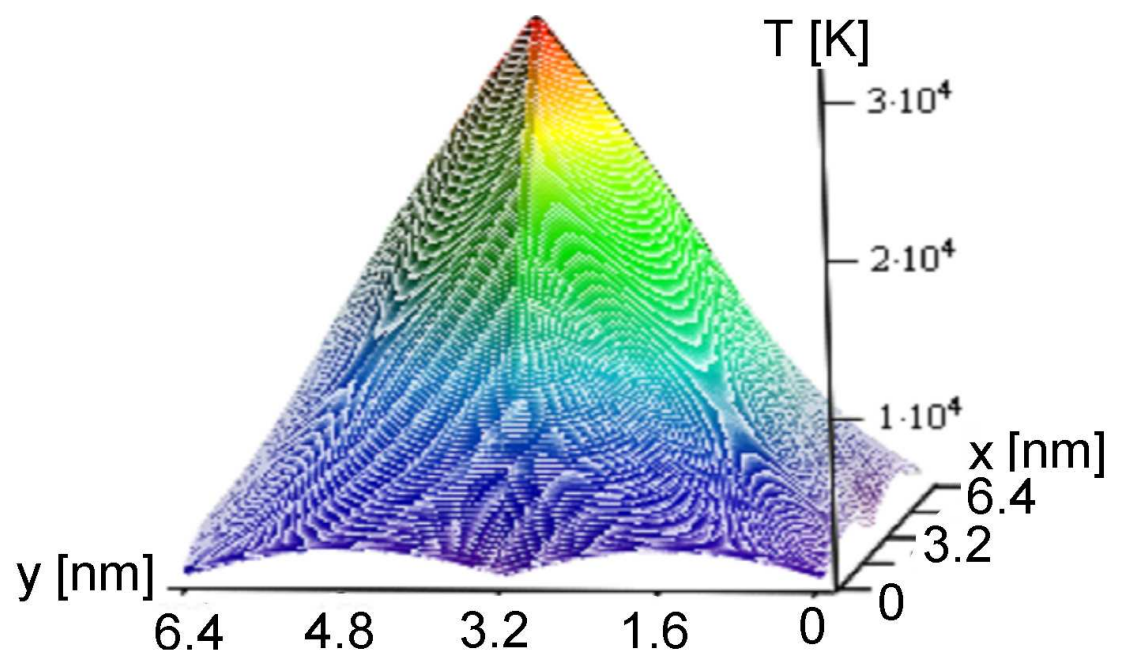

Fig. 1. Dependence of electron temperature on distance from the heating center after the first stage of ballistic thermodiffusion. Maximum distance from the irradiation center $R_{0}=3.21 \mathrm{~nm}$.

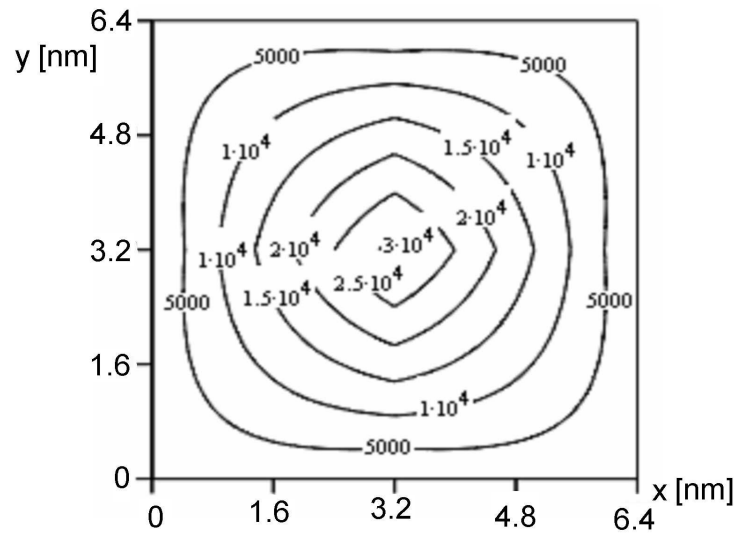

Fig. 2. Dependence of electron temperature on distance from the heating center after the first stage of ballistic thermodiffusion on the plane. Maximum distance from the irradiation center $R_{0}=3.21 \mathrm{~nm}$. 
In the beginning of the second stage, the electrons, exceeded by laser irradiation, are quickly diffusing and transferring their energy to the electrons of conductivity band [10]. This situation can be analyzed in terms of relaxation time $\tau$ approximation $[12,15,16]$. At the first moment of this relaxation stage, when electrons energies significantly exceed thermal energies of equilibrium electrons, but have the energies below the Fermi energy, are scattered by atoms and equilibrium electrons, generating the new nonequilibrium electrons system with much lower excess energy [17]. This moment before relaxation stage was named the "pretemperature" stage [18]. Solutions of nonlinear thermodiffusion equation for the second stage can be obtained using solutions for the nonlinear many-staged diffusion presented in the paper [11]. For the second stage we have the following solution:

$$
T_{2}\left(x, y, t_{2}\right)=\left(T_{\mathrm{s} 1}-T_{\mathrm{a}}\right) F\left(t_{2}\right) f\left(x_{1}, y_{1}\right), \quad F\left(t_{2}\right)=\frac{\left(D_{1} t_{1}\right)^{1 / 3}}{\left(D_{1} t_{1}+D_{2} t_{2}\right)^{1 / 3}},
$$

where $\sqrt{D t}$ in $f\left(x_{1}, y_{1}\right)$ expression (19) must be changed by $\left(D_{1} t_{1}\right)^{1 / 6}\left(D_{1} t_{1}+\right.$ $\left.D_{2} t_{2}\right)^{1 / 3}$. After the second thermodiffusion, the electrons must be de-excited and we must require that distance, which can be achieved by electrons, must be proportional to $\sqrt{t_{2}}$ and the thermodiffusion coefficient can be defined from (14) when $\alpha=1$ :

$$
D_{2}=\frac{x_{0}^{2}}{0.632^{2} t}, \quad x_{0}=v_{\mathrm{F}} \tau_{1}, \quad t=\tau_{1}, \quad D_{2}=6.902 \times 10^{3} \mathrm{~nm}^{2} / \mathrm{ps} .
$$

In the result of transition from ballistic movement to thermodiffusion these highly nonequilibrium electrons, for which the Fermi-Dirac distribution is not valid, after few collisions with atoms of lattice and equilibrium electrons generate the new nonequilibrium electrons in the system [17] but with lower excess energy of electrons. We can suppose that for these two states of excited nonequilibrium electrons the Boltzmann distribution and formula (5) are valid. For this nonequilibrium stage of electrons with lower energy [17], we can apply the obtained relaxation time $\tau_{2}=0.12 \mathrm{ps}$ [12]. Probability of subsequent collisions is lower by an order of magnitude [17] and relaxation time for transition from nonequilibrium state with lower energy to equilibrium by secondary collisions is much greater [10], $\tau_{3}=7 \mathrm{ps}$. For this time interval electron-phonon coupling constant $g$ must be included. The modified thermodiffusion coefficient dependence on the time must be defined using the Fermi-Dirac distribution for thermal electrons.

For transition from upper nonequilibrium excited state to lower nonequilibrium excited state, we have the following depending on time thermodiffusion coefficient:

$$
D_{2 \mathrm{t}}=\frac{D_{2}}{\alpha}\left(\frac{t_{2}}{\tau_{2}}\right)^{\alpha-1}, \quad \alpha=\frac{D_{2}}{D_{1}}, \quad \alpha=0.2998, \quad \tau_{2}=0.12 \mathrm{ps} .
$$

After substituting the following parameters for second thermodiffusion stage into (27): 


$$
\begin{aligned}
& D_{1}=2.30 \times 10^{4} \mathrm{~nm}^{2} / \mathrm{ps}, \quad t_{1}=\tau_{1}, \quad \tau_{1}=1.118 \times 10^{-15} \mathrm{~s}, \\
& T_{\mathrm{s} 1}=3.171 \times 10^{4} \mathrm{~K}, \quad \mathrm{~T}_{\mathrm{a}}=300 \mathrm{~K}, \quad \tau_{2}=\mathrm{t}_{2}=0.12 \mathrm{ps}
\end{aligned}
$$

from Eqs. (19), (27) we obtained temperature profiles for excited electrons. We presented these profiles like three-dimensional and two-dimensional pictures in Fig. 3 and Fig. 4.

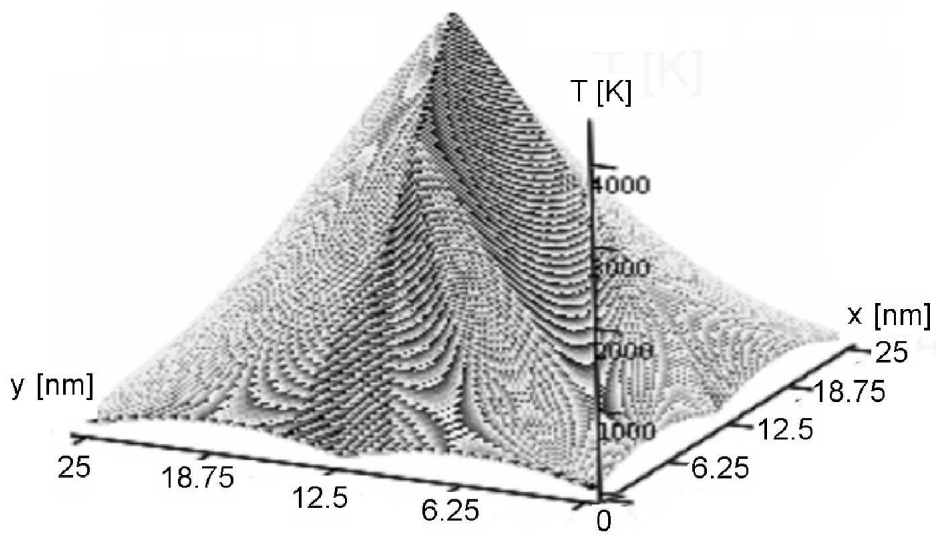

Fig. 3. Dependence of electron temperature on distance from the heating center after the second stage of thermodiffusion. Maximum distance from the irradiation center $R_{0}=12.5 \mathrm{~nm}$.

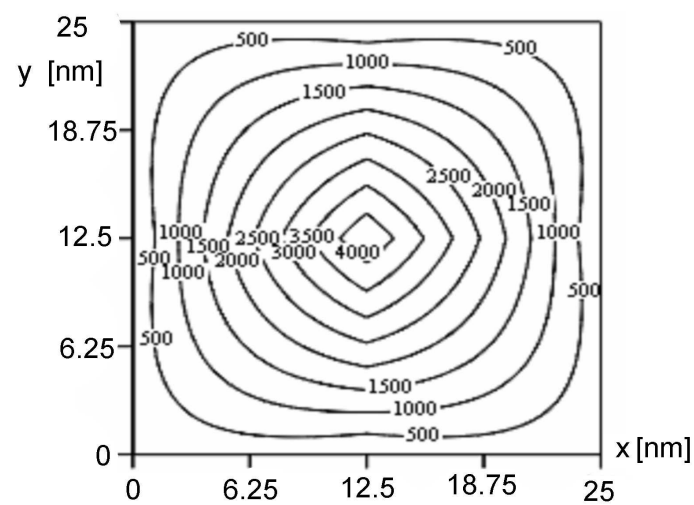

Fig. 4. Dependence of electron temperature on distance from the heating center after the second stage of thermodiffusion on the plane. Maximum distance from the irradiation center $R_{0}=12.5 \mathrm{~nm}$.

The maximum values of difference between hot electron temperature and lattice temperature $T_{2}\left(0,0, t_{2}\right)$, as well as radii $R\left(t_{2}\right)$ in the plane, shown graphically in Figs. 1-4 can be presented numerically as follows: 


$$
\begin{aligned}
& T_{2}(0,0,0.03 \mathrm{ps})=5.07 \times 10^{3} \mathrm{~K}, \quad R(0.03 \mathrm{ps})=10.9 \mathrm{~nm}, \\
& T_{2}(0,0,0.06 \mathrm{ps})=4.74 \times 10^{3} \mathrm{~K}, \quad R(0.06 \mathrm{ps})=11.6 \mathrm{~nm}, \\
& T_{2}(0,0,0.12 \mathrm{ps})=4.42 \times 10^{3} \mathrm{~K}, \quad R(0.12 \mathrm{ps})=12.5 \mathrm{~nm}, \\
& T_{2}(0,0,0.5 \mathrm{ps})=3.83 \times 10^{3} \mathrm{~K}, \quad R(0.50 \mathrm{ps})=14.4 \mathrm{~nm} .
\end{aligned}
$$

The obtained results show that, by using nonlinear thermodiffusion equation, we obtained thermal inertia phenomenon like in the paper [12], where the hyperbolic heat conduction equation was used.

Using (25) in conjunction with above presented hot electrons thermodiffusion parameters we can calculate energies of excited electrons after second thermodiffusion stage [11] or first stage of relaxation [17]:

$$
\begin{array}{ll}
Q(0.03 \mathrm{ps})=1.12 \times 10^{-15} \mathrm{~J}, & Q(0.06 \mathrm{ps})=1.12 \times 10^{-15} \mathrm{~J}, \\
Q(0.12 \mathrm{ps})=1.13 \times 10^{-15} \mathrm{~J}, & Q(0.5 \mathrm{ps})=1.14 \times 10^{-15} \mathrm{~J} .
\end{array}
$$

The presented results show that during the relaxation time interval $\tau_{2}=$ $0.12 \mathrm{ps}$ we have the thermodiffusion of electrons with constant energy, because electron-phonon interaction was not included. But we obtained smaller electron's energy than was introduced by laser irradiation. The transition from the ballistically heat transport to the second stage of thermodiffusion is connected with the reduction of electrons energies from (21) $E=3.20 \times 10^{-15} \mathrm{~J}$ to (32) $Q(0.03 \mathrm{ps})=1.12 \times 10^{-15} \mathrm{~J}$ and thermodiffusion coefficients decrease from (26) $D_{1}=2.302 \times 10^{4} \mathrm{~nm}^{2} / \mathrm{ps}$ to $(26) D_{2}=6.902 \times 10^{3} \mathrm{~nm}^{2} / \mathrm{ps}$. At the same time, electrons transfer part of their energy $\Delta E=E-Q=2.08 \mathrm{~J}$ to the ions of lattice.

\section{Conclusions}

The model of nonlinear diffusion [1-3] in the excited systems [11, 5] was applied to thermodiffusion of hot conductivity electrons in thin metal films irradiated by laser. Our results qualitatively coincide with the thermal inertia phenomenon obtained by solving one-dimensional hyperbolic heat conduction equation. In the paper [11] it was shown that diffusion coefficient is directly proportional to the population of the excited state. If population of excited states is decreasing the diffusion or thermodiffusion coefficients are also decreasing by time and diffusing particles are losing part of their energy. Thermal energy and temperature is localized as a result of thermal energy spreading by thermodiffusion process with decreasing by time thermodiffusion coefficient. In this case thermal energy introduced by the ballistic thermal conductivity is too large for spreading by thermodiffusion and some inertia of local concentration of energy can be obtained [19]. Results of our modeling qualitatively coincide with the fact that, when irradiation time of thin film of metal is about $0.4 \mathrm{ps}$, about half of irradiated energy is transmitted to the lattice [20]. 


\section{References}

[1] A.J. Janavičius, Phys. Lett. A 224, 159 (1997).

[2] A.J. Janavičius, A. Poškus, Acta Phys. Pol. A 107, 519 (2005).

[3] A.J. Janavičius, V. Stukaite, D.J. Zanevičius, Electron Tech. Ser. 2: Semiconductor Dev. 160, 27 (1983) (in Russian).

[4] S.M. Park, J.M. Kim, H.Ch. Lee, Ch.K. Kim, Jpn. J. Appl. Phys. 35, 1554 (1996).

[5] A.J. Janavičius, Ž. Norgèla, R. Purlys, Eur. Phys. J. Appl. Phys. 29, 127 (2005).

[6] A.J. Janavičius, Acta Phys. Pol. A 93, 505 (1998).

[7] A.J. Janavičius, Ž. Norgèla, R. Purlys, Acta Phys. Pol. A 104, 459 (2003).

[8] S.I. Anisimov, B.L. Kapeliovich, T.L. Perel'man, Sov. Phys.-JETP 39, 375 (1974).

[9] J.M. Ziman, Principles of the Theory of Solids, Cambridge University Press, Cambridge 1972, p. 472.

[10] C. Schafer, H.M. Urbasek, L.V. Zhigilei, Phys. Rev. B 66, 115404 (2002).

[11] A.J. Janavičius, Acta Phys. Pol. A 93, 731 (1998).

[12] J. Marciak-Kozlowska, Lith. J. Phys. 35, 616 (1995).

[13] A.J. Janavičius, S. Turskienè, Acta Phys. Pol. A 108, 979 (2005).

[14] A.J. Janavičius, Ž. Norgèla, R. Purlys, Acta Phys. Pol. A 104, 459 (2003).

[15] A.V. Chaplik, Zh. Eksp. Teor. Fiz. 60, 1845 (1971) [Sov. Phys.-JETP 33, 997 (1971)].

[16] G.F. Giuliani, J.J. Quinn, Phys. Rev. B 26, 4421 (1982).

[17] R.N. Gurzki, A.I. Kopieliovich, A.N. Kalinnenko, A.V. Yanovsky, E.N. Bogachek, Uzi Landman, H. Buchmann, L.W. Molekamp, Phys. Rev. B 68, 165318 (2003).

[18] R.N. Gurzhi, A.N. Kalinenko, A.I. Kopieliovich, Low Temp. Phys. 23, 44 (1997).

[19] A.J. Janavičius, G. Lūža, D. Jurgaitis, Acta Phys. Pol. A 105, 475 (2004).

[20] B. Rethfeld, A. Kaiser, M. Vicanek, G. Simon, Appl. Phys. A 69 [Suppl.], S109 (1999). 